\title{
Claves para el diseño de propuestas educativas intergeneracionales a partir de una experiencia de actividad física
}

\section{Keys to the design of intergenerational educational proposals from an experience of physical activity}

RAQUEL BECERRIL-GONZÁLEZ

Facultad de Educación de Palencia. Universidad de Valladolid. España raquel.becerril@uva.es

NiCOLÁs BORES-CALLE

Facultad de Educación de Palencia. Universidad de Valladolid. España nicolasjulio.bores@uva.es

ORCID: https://orcid.org/0000-0002-6720-0459

Recibido: 02-07-2019. Aceptado: 15-12-2019.

Cómo citar / Citation: Becerril-González, R. y Bores-Calle, N. (2019). Claves para el diseño de propuestas educativas intergeneracionales a partir de una experiencia de actividad física, Ágora para la Educación Física y el Deporte, 21, 111-124

DOI: https://doi.org/10.24197/aefd.0.2019.111-124

Resumen. El objetivo del trabajo es ofrecer unas claves para el diseño de propuestas educativas intergeneracionales. Durante 8 ediciones consecutivas hemos desarrollado una "actividad intergeneracional de orientación deportiva" y hemos recogido datos a partir de la observación participante y de entrevistas abiertas. Entre los resultados obtenidos cabe mencionar la existencia de muchas resistencias y dificultades a la hora de relacionarse entre generaciones; la inclinación a reunirse entre iguales y en familia; la tendencia a acabar realizando actividades con sesgo gerontológico; la constatación de que no todas las relaciones intergeneracionales son ideales; la conveniencia de que los participantes sean conscientes de las intenciones de la educadora; $y$, en fin, que relacionarse inter-generacionalmente es algo más complejo que juntar a personas de diferentes edades. A partir de estos datos, ofrecemos doce claves a tener en cuenta a la hora de planificar, desarrollar y evaluar actividades educativas que nos permitan construir una sociedad para todas las edades.

Palabras clave. Intergeneracionalidad; educación; actividades físicas.

Abstract: The purpose of this work is to offer some key ideas for the design of intergenerational educational proposals. During 8 consecutive editions we have developed an "intergenerational orienteering activity" and we have collected data from participant observation and open interviews. Data collected suggest that there are many resistances and difficulties when relating 
between generations; the inclination to meet peers and family; the tendency to organize activities with a gerontological bias; the confirmation that not all intergenerational relationships are ideal; the relevance of participants' awareness of the intentions of the educator; and that intergenerational relationships are more complex than merely bringing people of different ages together. From these data, we offer twelve keys ideas to take into account when planning, developing and evaluating educational activities that allow us to build a society for all ages.

Keywords: Intergenerationality; education; physical activities.

\section{INTRODUCCIÓN}

La edad cronológica actúa como uno de los referentes organizativos de la sociedad en la que nos desenvolvemos, operando como un referente central de la identidad de los individuos (Becerril, 2015, 19). De mantenerse las tendencias demográficas actuales, la esperanza de vida de un mayor número de personas en Occidente será cada vez más alta (INE, 2017).

Una sociedad más longeva es, a su vez, una sociedad en la que las distintas generaciones (Donati, 1999, 32) tienen que convivir un mayor número de años. Siendo así, desde el ámbito educativo debemos investigar las posibilidades de las nuevas formas de interacción entre las generaciones, tanto en el contexto familiar como en el comunitario y en la sociedad en general.

Se afirma que "tengamos conciencia generacional o no, las relaciones intergeneracionales no constituyen una elección, son consustanciales a lo humano [...]. Y durante todo nuestro recorrido biográfico nos cruzamos obligatoriamente con otros grupos generacionales" (IMSERSO, 2011, 578). Sin embargo, algunos de los problemas de la sociedad actual son el distanciamiento e incomunicación entre distintas generaciones, dificultando en ocasiones la convivencia y la cohesión social (Martínez, Moreno, y Escarbajal, 2017, 148).

Teniendo en cuenta que las distintas etapas vitales son construcciones socio-históricas, las características, connotaciones, prejuicios y estereotipos asignados a cada una de ellas también suelen marcar las actuaciones educativas destinadas a cada grupo de edad. En nuestro entorno, el trabajo socioeducativo se apoya en discursos contradictorios que, por un lado, hablan de participación social e intergeneracionalidad $\mathrm{y}$, por otro, reproducen $\mathrm{y}$ difunden propuestas puntuales y de ocio entre iguales. 
Se ha comprobado que, a medida que envejecemos, las relaciones sociales se reducen y que la mayoría de las relaciones intergeneracionales se suceden en familia (Becerril, Bores, y Rey, 2017).

Entendemos las relaciones intergeneracionales como un planteamiento sociopolítico y educativo clave para mejorar las relaciones interpersonales dentro de cualquier colectivo social (Friedman, 1999; Fundación PFIZER, 2015), alejándonos de otros planteamientos con el sesgo gerontológico que normalmente va asociado a este tipo de experiencias (Sánchez, 2015).

Muchas son las investigaciones que apuntan a los beneficios físicos, cognitivos y sociales de los contactos, las experiencias y los programas intergeneracionales (Hatton-Yeo \& Ohsako, 2001, 4-5; Martínez, Escarbajal, y Moreno, 2012; López, González, Sánchez, 2015; Martínez, Moreno, y Escarbajal, 2017). Pero no queremos caer en la peligrosa retórica que sostiene que todo lo que suene a relación intergeneracional es bueno. En esta ocasión nos interesan también las dificultades con las que nos encontramos a la hora de desarrollar propuestas educativas intergeneracionales.

A su vez, coincidimos con el sociólogo Mariano Sánchez (2015) en que no todas las relaciones entre generaciones son beneficiosas para las personas implicadas. Y añadimos que las relaciones intergeneracionales no tienen que ser (ni son) siempre positivas, ideales ni idílicas. Todo lo contrario: las relaciones intergeneracionales son, a priori, escasas y complejas.

A pesar de la importancia de la temática, hay que reconocer que muchas experiencias de educación intergeneracional no son suficientemente conocidas, analizadas y divulgadas. Los efectos de este tipo de educación han sido escasamente explorados y es preciso que comiencen a convertirse en objeto de investigación.

Precisamente en nuestra práctica laboral cotidiana la necesidad de alentar las relaciones entre generaciones (ONU, 2002) se ha convertido en una constante. En esta ocasión, investigamos sobre una de nuestras propuestas educativas con el objetivo de ofrecer algunas claves a tener en cuenta a la hora de realizar propuestas educativas intergeneracionales.

Para ello, partimos del análisis de una experiencia de educación social denominada "Actividad intergeneracional de orientación deportiva”, que se desarrolla anualmente en la localidad de Magaz de Pisuerga (Palencia-España). 


\section{Metodología}

Enmarcado en las programaciones de educación social, se organiza una práctica que llamamos "Actividad intergeneracional de orientación deportiva”. Básicamente, consiste en una mañana en la naturaleza haciendo un recorrido de orientación por parejas, compuestas por miembros de una diferencia mínima de 15 años (lo que se establece oficialmente como salto de generación). Se organiza en la localidad de Magaz de Pisuerga (Palencia-España) y la participación es abierta.

La mayoría de participantes residen en dicha localidad, destacando que, según la pirámide de población del municipio, la mayoría de sus habitantes se encuentran en la franja de entre los 10 y los 40 años. Un porcentaje amplio corresponde a personas que participan en otros proyectos de la localidad: infancia, apoyo escolar, "Huertos educativos", "Abrapalabra” (programa socioeducativo con personas adultas), juventud, asociacionismo, etc.

Además, también acuden participantes residentes en la capital y en otras poblaciones de la provincia, así como el alumnado de la Facultad de Educación de Palencia (Universidad de Valladolid) -concretamente de las asignaturas "La actividad física en la educación social", "Educación física adaptada a diferentes colectivos" y "Educación física en el medio natural”, correspondientes a los Grados de Educación Social y Magisterio-, y miembros de diferentes edades del Club ORCA (Orientación Río Carrión) de Palencia.

La investigación se estructura en varias fases. En la tabla siguiente (Tabla I) se resumen las ediciones, los instrumentos de recogida de datos que hemos utilizado cada vez y el número de participantes.

(Tabla I, página siguiente) 
Tabla I. Esquema del proceso de investigación seguido

\begin{tabular}{|c|c|c|}
\hline $\begin{array}{l}\text { Año (A partir } \\
\text { de fecha de } \\
\text { realización) }\end{array}$ & $\begin{array}{l}\text { Instrumento principal de recogida } \\
\text { de datos }\end{array}$ & Número de participantes \\
\hline $27 / 10 / 2012$ & $\begin{array}{c}\text { Observación no sistematizada de la } \\
\text { actividad }\end{array}$ & 36 \\
\hline 19/10/2013 & $\begin{array}{l}\text { Observación participante y externa de } \\
\text { la actividad }\end{array}$ & 62 \\
\hline $11 / 10 / 2014$ & $\begin{array}{l}\text { Observaciones sobre las llegadas, } \\
\text { inicio y explicaciones de la actividad } \\
\text { (ANTES) }\end{array}$ & 83 \\
\hline $18 / 10 / 2015$ & $\begin{array}{l}\text { Observaciones del comportamiento } \\
\text { de las parejas en el desarrollo de la } \\
\text { actividad (DURANTE) }\end{array}$ & 98 \\
\hline $16 / 10 / 2016$ & $\begin{array}{l}\text { Observaciones sobre los regresos de } \\
\text { la actividad y cierre (DESPUÉS) } \\
\text { Primeras entrevistas a participantes: } \\
\text { orales y escritas (menores) }\end{array}$ & 103 \\
\hline 29/10/2017 & $\begin{array}{l}\text { Entrevistas al finalizar la actividad } \\
\text { (DATOS A CORTO PLAZO. } \\
\text { IMPRESIONES INMEDIATAS) }\end{array}$ & 134 \\
\hline $\begin{array}{c}\text { Proceso } \\
\text { durante } 2018\end{array}$ & $\begin{array}{l}\text { Entrevistas abiertas pasado un tiempo } \\
\text { (DATOS A LARGO PLAZO) }\end{array}$ & $\begin{array}{l}\text { A partir de la selección de } \\
\text { participantes de la edición } \\
\text { del año 2017, habiendo } \\
\text { participado en varias } \\
\text { ediciones y/o propuestas de } \\
\text { Educación Social }\end{array}$ \\
\hline
\end{tabular}

(Fuente: Elaboración propia.)

La muestra está formada por las personas que han participado en cada edición de la actividad intergeneracional, atendiendo a diferentes criterios: edad, sexo, procedencia, experiencia en orientación y tipo de agrupación (familiar, comunitaria, entre iguales o asignada por la educadora).

Teniendo en cuenta los objetivos de la investigación, se emplean diferentes instrumentos y técnicas cualitativas (Tójar, 2006), fundamentalmente la observación durante el desarrollo de la jornada, así como entrevistas aleatorias a diferentes participantes.

En el contexto de cada jornada, tras informar a las personas participantes de los objetivos del estudio, se recogieron hechos, situaciones y conversaciones según los criterios de observación previamente establecidos, y teniendo en cuenta la totalidad del contexto en el que se suceden. 
Las primeras ediciones, basadas en una observación no sistemática, nos sirvieron para convencernos de la necesidad y posibilidad de investigar nuestra propia práctica, dando paso a la observación participante de la educadora y a observaciones externas por parte de los alumnos del Grado de Educación Social de la Facultad de Educación de Palencia como método de recogida de datos. En cada una de las fases, el estudio se centró en diferentes momentos de la práctica educativa investigada: llegadas de participantes, acogida, espacios, agrupaciones, momentos previos a la actividad por el medio natural, explicaciones e inicios (antes); observaciones de parejas/grupos durante los recorridos, tomas de decisiones, formas de comunicación, cómo se establecen relaciones (durante); llegada, recuento de puntos, cierre de la actividad, tipo de agrupaciones y relaciones (después).

La recogida de datos se complementó con entrevistas a los participantes. Se pretendía obtener impresiones inmediatas y espontáneas sobre la actividad y el tipo de relaciones que se daban durante la misma. Para ello, nos pareció una buena pregunta: ¿cómo evalúas la actividad, en cuanto a la relación con tu compañero/a?”. Todo ello fue grabado en audio de forma anónima y anotando la edad y el sexo.

Una vez recogidos los datos se procedió a su reducción, análisis y categorización, tratando de teorizar (Goetz y LeCompte, 1988, 167). Investigando desde la práctica, en lugar de describir la actividad, tratamos de analizar sus posibilidades y dificultades, centrándonos en las relaciones entre participantes de edades diferentes con el fin de detectar algunas de las claves a tener en cuenta para potenciar la intergeneracionalidad de las propuestas educativas.

\section{RESULTADOS E INTERPRETACIÓN DE LOS DATOS}

\subsection{Resistencias y dificultades}

A pesar de ser una actividad cuidadosamente diseñada para potenciar las relaciones intergeneracionales, los datos recogidos mediante la observación, los registros anecdóticos o las entrevistas, muestran las dificultades existentes para establecer verdaderas relaciones intergeneracionales.

Participan personas de varias generaciones, juntos en el mismo espacio, pero las relaciones horizontales y en condiciones de igualdad y 
cooperación son escasas. Los comentarios recogidos en los instantes previos a la actividad son muestra de ello:

- [SARA, 14 años]: A la orientación... ¿¿nos podríamos apuntar Paula [16 años] y yo juntas? Es que todavía no sabemos si vamos a apuntarnos o no y no tenemos pareja...

- [EDUCADORA SOCIAL]: Sabéis que no... Lo de la pareja no es problema, yo os la busco.

- [PAULA, 16 años]: (Risas) Es que... no queremos ir con alguien... (risas).

- [SARA]: Es que nosotras si vamos a ir juntas nos apuntamos. Si no, no es que tengamos muchas ganas. [Registro "Actividades juveniles", $12 / 10 / 2016]$

En el ejemplo, podemos observar una muestra del tipo de resistencias que nos encontramos en todas las ediciones. En este caso, al ser personas que participan en otros programas, lo comentan con la educadora en tono de humor, pero mostrando incomodidad al imaginarse en una situación que rompe con la tendencia de estar entre iguales. Algunas participantes tratan de evitar el requisito de participación junto a personas con una diferencia de edad de, al menos, 15 años: "Yo iré con mi marido, que para un día que podemos... [Se refiere a que él ese día no trabaja]. Además... ique tampoco sabemos a quién decir que venga con nosotros!" (Silvia, 59 años). Este tipo de datos los interpretamos como pruebas de que las relaciones habituales y normalizadas con personas de diferentes edades son escasas. Aunque somos conscientes de que hay diversidad de motivos para preferir la participación con iguales, a nosotros nos importan las resistencias para estar con los de otras edades.

\subsection{Tendencia social: entre iguales y en familia}

Analizando los datos de inscripción y de las observaciones, hemos comprobado que durante la actividad se tiende a ir en familia. Además, durante el recorrido se agrupan entre iguales:

Nos hemos juntado con otra familia, y menos mal, porque los padres tenían algo más de experiencia y nos han ayudado, si no... no habíamos llegado. (Juan, 45 años y Maite, 41 años) 
Relatando en las entrevistas variadas justificaciones sobre los motivos:

[Al principio] Hemos ido con las mamás. Los papás iban demasiado rápido y nos perdían (risas). Al final hemos acabado nosotros solos. (Julián, 12 años; Mario, 11 años; Ander, 13 años, Ainhoa, 11 años).

Este hecho provoca gran número de interacciones sociales, valoradas como compañerismo, muestras de diversidad o una forma de ayudar:

Compañerismo, porque hemos ido con más gente y hemos decidido conjuntamente. (Pedro, 43 años y María, 11 años)

Hemos ido con otra pareja a ratos, ayudándonos. (Jesús, 35 años; Alberto, 8 años)

Sin embrago, poniendo el foco en los objetivos de la investigación, resta posibilidades en la toma de decisiones intergeneracionales.

\subsection{Se evita el sesgo gerontológico}

En nuestro contexto, la tendencia es a trabajar por colectivos configurados por cuestiones de edad. Las propuestas educativas intergeneracionales tienden a ser puntuales, asociadas con propuestas que juntan a grupos de infancia con personas mayores (abuelos-nietos), normalmente en espacios diseñados para los unos o los otros: escuelas, residencias, centros de día y locales municipales. Se dice que esto produce numerosos beneficios (diferentes para una generación y la otra), por el simple hecho de estar juntos.

Esta investigación permite comprobar que nuestra actividad elude el sesgo gerontológico (Sánchez, 2015), puesto que las edades de los participantes, parejas conformadas por personas de diferentes edades del ciclo vital (desde meses hasta 73 años), superan el clásico de juntar mayores e infancia. Algo que también se refleja en los espacios utilizados: el medio natural no está asignado, a priori, a una generación u otra, permitiendo que los recorridos sean flexibles y adaptados para personas de cualquier edad y con un objetivo a perseguir claro $\mathrm{y}$ motivador para ambas generaciones:

Está adaptado para todos, venimos personas con edades muy diferentes e incluso con mascotas. (Beatriz, 37 años) 
Hice algo de orientación en la mili..., pero lo de venir de todas las edades y con los críos y así está muy bien. Muy bien. (Rubén, 40 años)

Además, los conocimientos necesarios para practicar orientación deportiva no se presuponen propios de una generación u otra:

El que sabe (de orientación) es él [su hijo de 9 años] que es el que ha estado contigo [a la Educadora. Se refiere a cursos de orientación]. (Andrés, 50 años)

Esto sí puede ocurrir en propuestas donde las herramientas son, por ejemplo, el uso de las tecnologías de la información y la comunicación (partiendo de que son los jóvenes los que enseñan y los mayores los que aprenden) o en dinámicas basadas en las tradiciones: juegos populares, canciones, narraciones (pensando que los mayores son los que tienen que trasmitirlo).

\subsection{Las relaciones intergeneracionales no son siempre ideales}

Se parte de que la intergeneracionalidad no tiene que responder siempre a patrones de éxito. Por el contrario, consideramos que tras un análisis siempre positivo e idealizado de las relaciones entre personas de distintas edades puede esconderse cierto paternalismo hacia las mayores. Pareciera que, al hacer actividades con personas de diferentes edades, las generaciones más jóvenes hacen el favor de dedicar tiempo a las mayores. Y sólo por ello, ya es bueno, tierno, emocional o las relaciones interpersonales fluyen por sí mismas.

Apostamos por romper con esas ideas estereotipadas, al mismo tiempo que reivindicamos que no todas las relaciones intergeneracionales tienen que ser positivas, beneficiosas, ni ideales. Lo importante es que sucedan, "[...] ya sean de consenso, de cooperación o de conflicto" (Sánchez et al., 2007, p. 42).

Fijándonos en las parejas que se mantienen durante la actividad, observamos que lo hacen de manera consciente: "Decidíamos las cosas los dos, aunque [...] he tenido que redirigir su interés hacia la actividad conmigo varias veces" (Luis, 38 años), y con dificultades: “[...] el momento que más me ha gustado es cuando el niño [de 4 años] ya se cansa y he tenido que buscar la estrategia para motivarlo y acabar juntos” (Jorge, 39 años). 
En estos casos, relatan (y se observan) discusiones, toma de decisiones, acuerdos, rupturas y diferencias:

Tenemos nuestros más y nuestros menos [...] Ha sido terapia padre e hija (risas). (Juan, 41 años y Esther, 14 años)

La relación la llevamos muy bien, pero en cuanto a la actividad depende de las capacidades, nos amoldamos bastante al niño. (Alí, 51)

Hemos dialogado. (Isabel, 62 años).

De este modo, podemos hablar de intergeneracionalidad también en realidades donde no existen siempre acuerdos entre generaciones o en contextos que visibilizan conflictos. Entendiendo el conflicto como algo común y necesario en las relaciones interpersonales, de cualquier tipo.

\subsection{Conocimiento de la finalidad de la actividad}

Actualmente coexisten más generaciones y la tendencia se mantendrá en el futuro. Aunque en apariencia nos cruzamos con personas de diferentes edades, las relaciones intergeneracionales reales y normalizadas son escasas. Por ello éstas suelen estar mediadas por propuestas educativas y/o de ocio que tienen entre sus objetivos potenciar la intergeneracionalidad.

Comprobamos que las personas que mejor conocen la finalidad de la actividad, han participado otros años y en otros proyectos educativos de la localidad, relatan más elementos relacionados con la intergeneracionalidad y lo valoran de forma positiva:

[...] es complicado decidir todo el tiempo juntos dónde vamos, Mohamen [13 años] está pendiente de ver qué hacen los otros [participantes] y tengo que tener paciencia... [...] Eso es lo interesante, por eso aprendemos los dos. (José, 38 años)

De este modo persiguen el objetivo y son conscientes de las dificultades.

Los datos muestran la necesidad del trabajo de, en este caso, la educadora. En nuestro contexto se hace necesaria la intervención del profesional que impulse/fuerce un tipo de relaciones que no se dan de manera normalizada. Al vincular prácticas como la que se emplean en esta investigación, con otras programaciones socioeducativas y con una 
visión comunitaria, vemos los beneficios de potenciar las relaciones intergeneracionales y la necesidad de prolongarlas en el tiempo.

\subsection{Más que juntar gente de diferentes edades}

En definitiva, los datos sugieren que para que se establezcan relaciones intergeneracionales no es suficiente con juntar personas de diferentes edades en un mismo espacio. Sin embargo, demuestran que el diseño de la actividad la convierte, a corto plazo, en una experiencia atractiva y enriquecedora al potenciar el número de interacciones intergeneracionales con un objetivo claro, en un espacio flexible y con una propuesta inclusiva.

Es la cuarta o la quinta vez que participamos juntos, lo hemos pasado muy bien”. (Mohamed, 14 años)

Llevamos un niño de 5 años y ha corrido hasta Villamediana y eso es mucho". (Alí, 51 años)

La gente nos pregunta por experiencia, son muchos años participando". (Abilio, 69 años).

Sin embargo, es necesario seguir investigando las implicaciones intergeneracionales a largo plazo, para establecer los elementos educativos a tener en cuenta para que sea prácticas realmente transformadoras.

\section{EPÍLOGO. EL DISEÑO DE ACTIVIDADES INTERGENERACIONALES}

Con esta investigación, sin obviar sus limitaciones, hemos pretendido esbozar algunas claves a tener en cuenta a la hora de realizar propuestas educativas que potencien las relaciones intergeneracionales, pues éstas, además de un medio para determinados propósitos, constituyen un objetivo en sí mismas, alcanzable mediante medidas específicas (Fundación EDE, 2015). En este sentido, enumeramos algunas que consideramos fundamentales:

- Evitar, como suele ocurrir en nuestro contexto, que las relaciones intergeneracionales sean anecdóticas y superficiales. 
- Es difícil que se establezcan vínculos intergeneracionales mediante propuestas educativas puntuales 0 de carácter extraordinario.

- Una propuesta educativa y enriquecedora es más que juntar personas de diferentes edades.

- El tipo de actividad, la metodología y la acción del educador son fundamentales para potenciar las relaciones intergeneracionales.

- Los espacios influyen en el tipo de relaciones que se establecen.

- Las relaciones equitativas se provocan cuando hay un objetivo claro y motivador para ambas generaciones.

- Las relaciones intergeneracionales no tienen que ser siempre ideales ni positivas. Lo importante es trabajar para que sucedan.

- El conflicto forma parte de las relaciones y es necesario tanto para el proceso de aprendizaje como para la consecución del objetivo común.

- Evitar el sesgo gerontológico implicando al mayor número de generaciones.

- Partir de una visión comunitaria a la hora de programar actividades y proyectos intergeneracionales.

- Prolongar los programas y actividades para lograr relaciones intergeneracionales reales.

- Los participantes deben conocer los objetivos que se persiguen y sus dificultades, buscando la implicación total en el proceso educativo.

Con estas claves, teniendo en mente el objetivo último de construir una sociedad para todas las edades, visibilizamos parte de los resultados de nuestras investigaciones, que repercuten en la mejora de nuestra práctica, tratado de contribuir a la mejora de las realidades sociales y educativas. 


\section{BIBLIOGRAFÍA}

Becerril, R. (2015). Análisis de los hitos de consciencia del envejecimiento a partir de relatos de personas mayores. Tesis de doctorado. Universidad de Valladolid. Disponible en:

https://uvadoc.uva.es/bitstream/handle/10324/13190/TESIS697-

150731.pdf;jsessionid=C1D2E5A6625E6811D77D090DDF19252A?seque $\underline{\text { nce }=1}$

Becerril, R.; Bores, N.J.; Rey, V. (2017). Imagen corporal, envejecimiento y educación social. RES, Revista de Educación Social, 24. Recuperado en: http://www.eduso.net/res/admin/archivo/docdow.php?id=993 (el 7 de diciembre de 2018)

Donati, P. (1999). Familia y generaciones. Desacatos. Revista de Antropología Social, 2, 27-49

Friedman, B. M. (1999) Connecting generations: integrating aging education and intergenerational programs with elementary and middle grades curricula. Needham Heights, MA: Allyn y Bacon.

Fundación EDE. (2015). Hacia una sociedad intergeneracional. ¿Cómo impulsar programas para todas las edades? Bilbo: Diputación Foral de Bizkaia

Fundación PFIZER. (2015). Estudio sobre balance vital y relaciones intergeneracionales en España. Informe de resultados. Recuperado en: https://www.fundacionpfizer.org/sites/default/files/informe_balance_vital_ y_relaciones_intergeneracionales.pdf (el 8 de noviembre de 2018)

Goetz, J.P y LeCompte (1988). Etnografía y diseño cualitativo en investigación educativa. Madrid: Morata.

Hatton-Yeo, A. y Ohsako, T. (2001). Programas intergeneracionales: política pública e implicaciones de la investigación. Una perspectiva internacional. París: UNESCO.

IMSERSO (2011). Libro Blanco del Envejecimiento Activo. Madrid: Ministerio de Sanidad y Política Social, Secretaría General de Política Social y Consumo Instituto de Mayores y Servicios Sociales. 
Instituto Nacional de Estadística, INE (2017). Gráficos y tablas sobre esperanza de vida. Recuperado el 6 de junio de 2019 en: https://www.ine.es/ss/Satellite?L=es_ES\&c=INESeccion_C\&cid=1259926 $\underline{380048 \& p=1254735110672 \& \text { pagename=ProductosYServicios/PYSLayout }}$

López, M.T; González, V. y Sánchez, A.J. (2015). Personas mayores y solidaridad intergeneracional en la familia. El caso español. Madrid: Dykinson.

Martínez, S.; Escarbajal, A. y Moreno, P. (2012). El rol de los abuelos en la relación con sus nietos. Una aproximación cualitativa desde el punto de vista educativo. En G. Pérez (ed.) I Simposio Internacional de Envejecimiento Activo y Solidaridad Intergeneracional. Recurso audiovisual. Madrid: UNED.

Martínez, S.; Moreno, P. y Escarbajal, A. (editores) (2017). Envejecimiento activo, programas intergeneracionales y educación social. Madrid: Dykinson.

Organización de las Naciones Unidas, ONU (2002). Informe de la II Asamblea Mundial sobre envejecimiento. A/CONF.197/9. Nueva York: Naciones Unidas,

Sánchez, M. (2015). Hablamos de intergeneracionalidad con Mariano Sánchez. Consultado en: http://www.aitorurrutia.eu/2015/02/mariano sanchez/ (el 15-05-2017)

Sánchez, M. (Coord.). (2007). Programas intergeneracionales. Hacia una sociedad para todas las edades. Colección estudios sociales, 23. (Fundación La Caixa.) Obtenido el 15 de noviembre de 2017 en: https://www.imserso.es/InterPresent2/groups/imserso/documents/binario/0 c01newmanysanchezlacaixa.pdf

Tójar, J. C. (2006). Investigación cualitativa. Comprender y actuar. Madrid: La Muralla. 